\title{
Relaciones entre el inicio y el término de la estación lluviosa en Centroamérica y los océanos Pacífico y Atlántico tropical
}

\author{
Eric Alfaro ${ }^{1}$, Luis Cid ${ }^{2}$ y David Enfield ${ }^{3}$ \\ ${ }^{1}$ Departamento de Oceanografía, casilla 2407, Universidad de Concepción, Concepción, Chile \\ ${ }^{2}$ Departamento de Estadística, Universidad de Concepción, Concepción, Chile \\ ${ }^{3}$ NOAA Atlantic Oceanographic and Meteorological Laboratory, Miami, Florida
}

\begin{abstract}
RESUMEN. En los últimos años diversos estudios han mostrado que las anomalías en los campos de temperaturas de los océanos Pacífico y Atlántico Tropical están relacionadas con variaciones, tanto en la intensidad como en la duración de la estación lluviosa sobre Centroamérica. En este trabajo se usaron, como variables independientes, distintos índices oceanográficos y atmosféricos, principalmente de las distribuciones de la temperatura superficial del mar (TSM) en los océanos Pacífico y Atlántico Tropical, para explicar las variaciones en las fechas de inicio (IELL) y término (TELL) de estación lluviosa sobre la región centroamericana. El índice del Atlántico Norte (ATN) y los índices IOS-Niño3 mostraron las mayores correlaciones con el IELL y el TELL, respectivamente.
\end{abstract}

Palabras claves: Interacción océano-atmósfera; ENOS; Variabilidad en el Atlántico.

\section{Relationships between the Start and End Date of the rainy season in Central America and the Tropical Atlantic and Pacific Oceans}

\begin{abstract}
In recent years, several studies had shown that anomalies in the sea surface temperature of the Tropical Atlantic and Pacific Oceans are related to variations in the intensity and timing of the rainy season in Central America. In order to study anomalous behavior of the rainy season over Central America, Tropical Atlantic and Pacific Oceans indices are used to produce correlation series with the starting and ending date (IELL and TELL) of the rainy season. The North Atlantic (ATN) and SOI-Niño3 indices, shown the main correlations with the IELL and the TELL respectively. Keywords: Ocean-Atmosphere Interaction; ENSO; Atlantic variability
\end{abstract}

\section{INTRODUCCIÓN}

A pesar de que la región centroamericana está rodeada al Este por el Mar Caribe y al Oeste por el Océano Pacífico (Fig. 1), el estudio de las anomalías de los campos de precipitación sobre la región, ha estado centrado básicamente en la influencia sobre ésta de las variaciones interanuales experimentadas por el Pacífico Ecuatorial Oriental, principalmente a través de estudios de correlaciones con El Niño-Oscilación del Sur (ENOS) (e.g. Aceituno, 1988; Díaz y Kiladis, 1993; Hastenrath, 1976, 1978; Kiladis y Díaz, 1989; Rogers, 1988; Ropelewski y Halpert, 1987, 1989, 1996).

Climáticamente, la Vertiente Pacífica de la región centroamericana es afectada durante el año por un período lluvioso y otro seco, ambos relacionados con el debilitamiento o reforzamiento de la intensidad del flujo alisio, inducido sobre la región por el anticiclón del Atlántico Norte y por la actividad convectiva asociada a la migración anual de la Zona de Convergencia Intertropical (ZCIT), ubicada, en promedio, entre $5-10^{\circ} \mathrm{N}$. Esta migración anual de la ZCIT hacia el norte, sucede durante la primavera boreal al final de abril y principios de mayo. Este patrón es interrumpido por períodos de precipitación reducida o «veranillos» entre julio y agosto (Ramírez, 1983), los cuales coinciden con una intensificación de los vientos alisios sobre el Caribe. Se produce un segundo pico de precipita- 
ción en octubre, generalmente mayor que el primero, debido al restablecimiento de las condiciones anteriores, que luego declina hacia un mínimo entre febrero y marzo, al emigrar la ZCIT hacia el sur. A diferencia de la Vertiente Pacífica, la Vertiente Caribe de la región centroamericana está dominada por la precipitación con nubosidad estratiforme asociada, producto de la interacción durante todo el año del flujo alisio y los Andes Centroamericanos, lo que provoca que en general, esta vertiente no presente una estación seca definida. Las desviaciones climáticas no estacionales en Centroamérica están
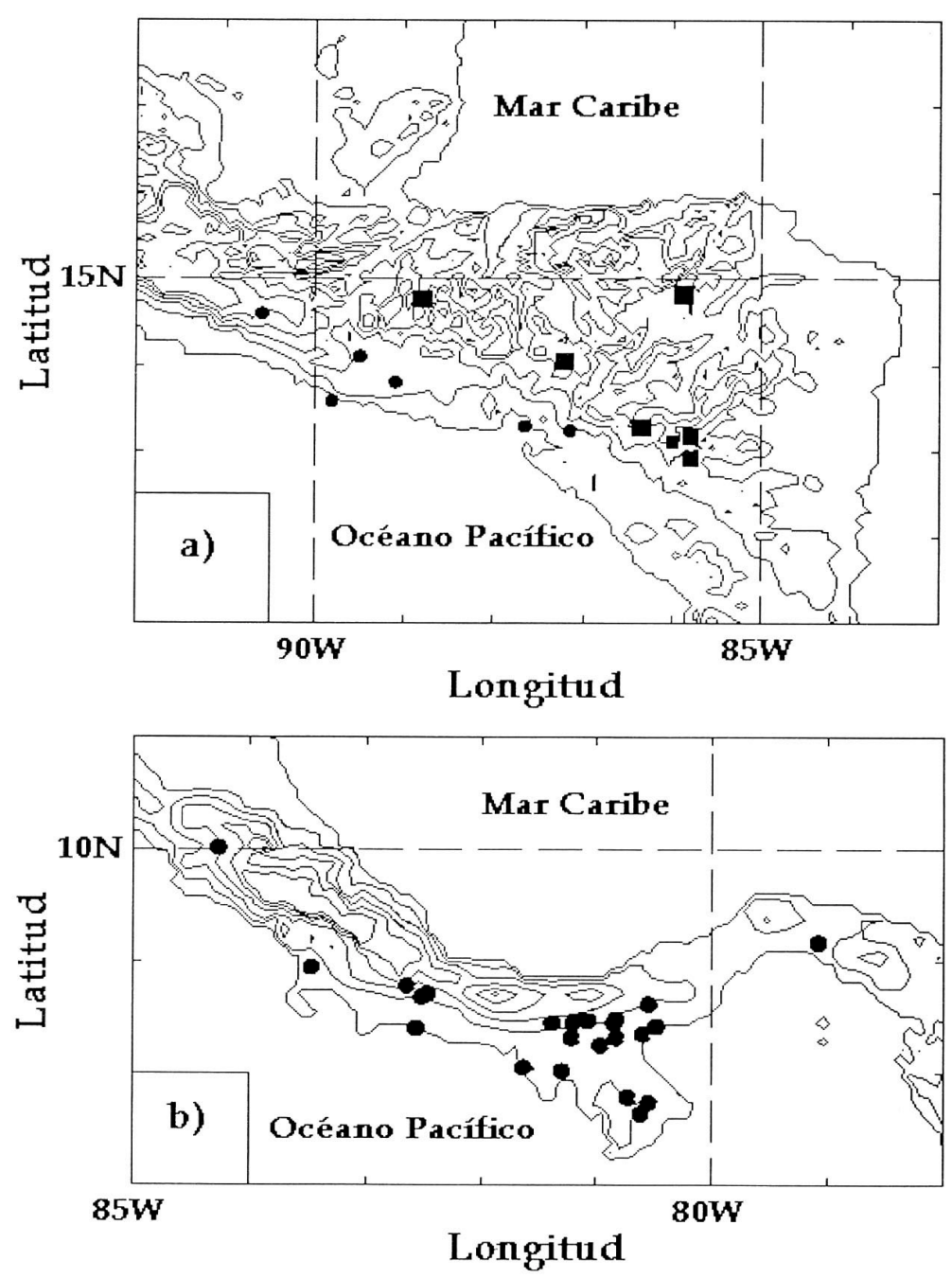

Figura 1. Estaciones usadas, los círculos sólidos indican estaciones sobre la Vertiente Pacífica y los rectángulos indican estaciones sobre la Vertiente Caribe, a) al norte de $11^{\circ}$ y b) al sur de $11^{\circ}$. Las líneas denotan los niveles topográficos de 300, 700, 1100, 1500, 1900 y $3000 \mathrm{~m}$.

Figure 1. Stations used, the filled dots are stations on the Pacific drainage and the rectangles are stations on the Caribbean drainage, a) north of $11^{\circ}$ and $b$ ) south of $11^{\circ}$. The lines are for $300,700,1100,1500,1900$ and 3000 m topographic levels. 
asociadas con movimientos meridionales anómalos de la ZCIT y fluctuaciones asociadas al régimen de vientos alisios del NE, la cual afecta el clima desde el sur de México hasta Panamá (Chacón y Fernández, 1985).

Durante el año, en la región del Caribe y Centroamérica, la evaporación media fluctúa entre los 5-8 mm por día (Gray, 1993). Podríamos decir entonces que la estación lluviosa sobre Centroamérica se establece cuando las necesidades hídricas de la región son satisfechas por la precipitación; esto es, cuando la cantidad de precipitación es superior a la evapotranspiración (Yao, 1981).

La región centroamericana tiene una fuerte asociación climática, a través de teleconecciones, con el Pacífico Ecuatorial Este y el Atlántico Tropical (Waylen et al., 1994). Lo anterior motivó que algunos autores empezaran a tomar en cuenta la influencia del océano Atlántico sobre los campos de precipitación en Centroamérica (e.g. Enfield, 1996; Enfield y Mayer, 1997; Waylen et al., 1994, 1996a, 1996b), pero, al igual que en los estudios anteriores, lo complicado de la geografía regional y la inexistencia de un banco de datos con una buena resolución espacial, ha causado que la relación entre la precipitación en Centroamérica y la Temperatura Superficial del Mar (TSM) del océano tropical, haya sido un parámetro difícil de cuantificar.

Este estudio pretende complementar los trabajos anteriormente mencionados, al cuantificar la influencia, de los Océanos Pacífico y Atlántico Tropical sobre el inicio (IELL) y el término (TELL) de la estación lluviosa en Centroamérica a través de un análisis de correlación cruzada. Para ello se utilizaron los índices propuestos por Enfield (1996) y un conjunto de 37 estaciones con registro pluviométrico diario sobre la región (Fig. 1). Este análisis permitió establecer el mes de máxima correlación entre los distintos índices y el IELL/TELL. Los detalles acerca de los índices usados y la obtención del IELL/ TELL se describen en la próxima sección de datos y metodología.

\section{DATOS Y METODOLOGÍA}

El área interés en el presente estudio es el istmo centroamericano, abarcando desde Guatemala y Belice en el norte, hasta Costa Rica y Panamá en el sur. Se usaron datos de acumulados de precipitación diaria para 37 estaciones de un conjunto original de 53 estaciones (Fig. 1). Los datos de precipitación fueron obtenidos del Centro de Investigaciones Geofísicas de la Universidad de Costa Rica y del Instituto Meteorológico Nacional de Costa Rica. Se obtuvo una buena cobertura de la Vertiente Pacífica de Centroamérica aunque no se pudo obtener datos de precipitación de registro diario para estaciones ubicadas sobre la Vertiente Pacífica nicaragüense.

Los índices del Atlántico Tropical y Niño3 fueron los mismos usados por Enfield (1996), debido a que ellos están asociados con áreas oceánicas que presentan alguna influencia sobre la precipitación en Centroamérica (e.g. Enfield, 1996; Ropelewski y Halpert, 1996). Para la construcción de estos índices, se utilizó un conjunto de datos de TSM $2^{\circ} \mathrm{x}$ $2^{\circ}$ elaborado por Smith et al., (1996), a partir del conjunto de datos COADS (Woodruff et al., 1987). Estos índices fueron calculados como el promedio de los puntos de la rejilla dentro de un área oceánica determinada. Las áreas del Atlántico Tropical Norte y Sur usadas fueron $22^{\circ} \mathrm{N}-6^{\circ} \mathrm{N}, 80^{\circ} \mathrm{W}-15^{\circ} \mathrm{W}$; y $2^{\circ} \mathrm{N}-22^{\circ} \mathrm{S}, 35^{\circ} \mathrm{W}-15^{\circ} \mathrm{E}$, respectivamente. El área usada para la construcción del índice Niño3 fue $6^{\circ} \mathrm{N}-6^{\circ} \mathrm{S}, 150^{\circ} \mathrm{W}-90^{\circ} \mathrm{W}$ (Fig. 2), este índice difiere un poco con la definición tradicional del índice Niño3 $\left(5^{\circ} \mathrm{N}-5^{\circ} \mathrm{S}, 150^{\circ} \mathrm{W}-90^{\circ} \mathrm{W}\right)$, pero la correlación entre ellos es de 0.97 (Enfield, 1996). Adicionalmente a estos índices oceánicos, se usó el Índice de Oscilación del Sur (IOS), el cual se toma como la presión estandarizada de Tahití menos la de Darwin (Ropelewski y Jones, 1987). Este fue obtenido del Climate Analysis Center de la NOAA, EUA. El período utilizado de estos índices y de los registros de precipitación, comprendió de enero de 1950 a diciembre de 1994.

El criterio de selección de las estaciones de precipitación fue que las mismas presentaran una marcada estación seca durante el año (menos de $5 \mathrm{~mm}$ acumulado de precipitación diario) y que su estación lluviosa se ubicara entre los meses de abril a noviembre (Fig. 3). Las estaciones descartadas no cumplieron con alguno de los criterios anteriores. Geográficamente, 7 de las estaciones estudiadas se ubicaron sobre la Vertiente Caribe, específicamente en el Caribe hondureño y nicaragüense no costero, mientras que 30 de ellas se ubicaron sobre la Vertiente Pacífica (Fig. 1). Lo anterior está de acuerdo con lo discutido en la introducción, pues en general, la Vertiente Pacífica es la que presenta una estación seca definida. 


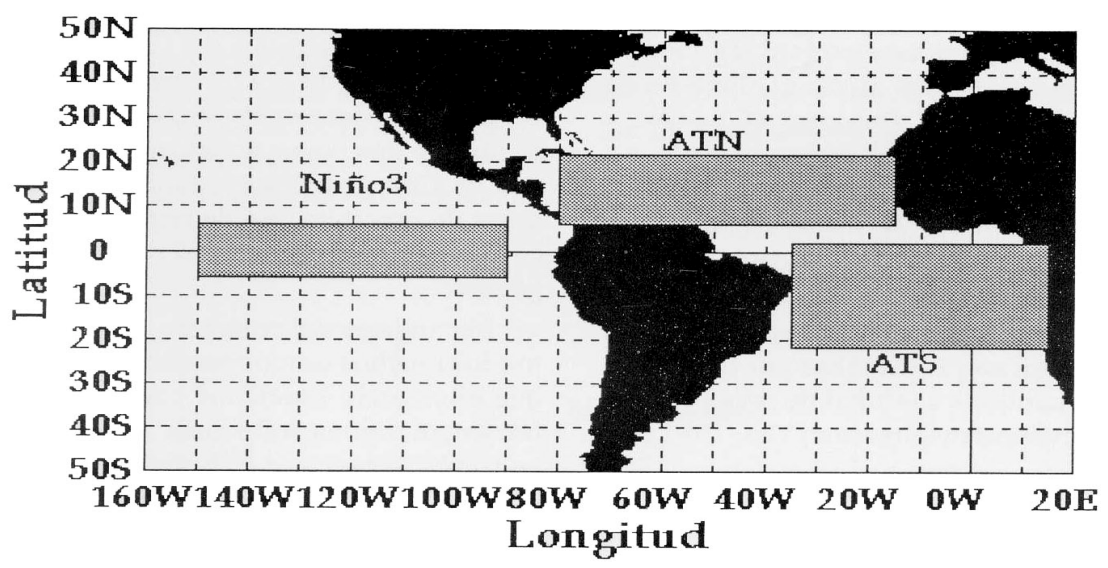

Figura 2. Areas oceánicas usadas para la construcción de los índices.

Figure 2. Oceanic areas used in the indices construction.

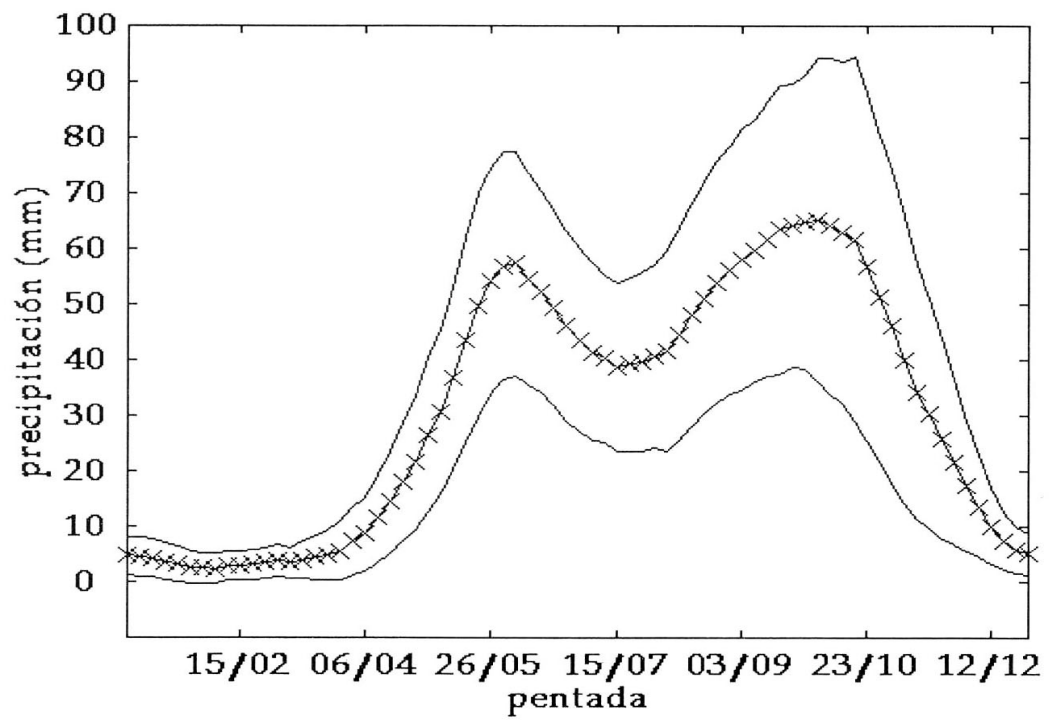

Figura 3. Ciclo anual promedio (línea con cruces) de la precipitación, para las estaciones usadas en la figura 1. Las bandas representan una desviación estándar. En el eje x, los valores representan las fechas del primer día de la pentada.

Figure 3. Rainfall annual mean cycle (cross line) of the gauge stations used in the figure 1. Bands are given for one standard deviation. In the $x$ axis, values represent the first pentad's day. 
Luego, el conjunto de datos de acumulados diarios fue reducido a pentadas (precipitación acumulada durante 5 días). El primer día del año que se usó para producir las pentadas fue el $1^{\circ}$ de enero. No se tomó en cuenta el día 29 de febrero de los años bisiestos, por lo que se obtuvo siempre 73 pentadas por año.

El criterio para el cálculo del inicio de la estación lluviosa (IELL) fue el siguiente:

- Se considera que la estación lluviosa se ha iniciado si la pentada en estudio tiene un valor de 25 $\mathrm{mm}$ o más ( $5 \mathrm{~mm} /$ día) de lluvia, al menos una de las dos pentadas siguientes (en orden creciente) también tiene(n) un valor de $25 \mathrm{~mm}$ o más de lluvia y que las pentadas anterior y posterior a esta última, tengan más de $0.5 \mathrm{~mm}$ de lluvia acumulada. Esto último, porque la resolución del instrumento es de $0.1 \mathrm{~mm}$, esta medida se reporta usualmente como traza de precipitación. Lo anterior se puede escribir como t = IELL sí: a) $x(t)^{3} 25 \mathrm{~mm} \wedge x(t+1){ }^{3} 25 \mathrm{~mm}$ $\wedge x(t+2)>0.5 \mathrm{~mm}, \mathrm{o}, \mathrm{b}) x(t){ }^{3} 25 \mathrm{~mm} \wedge x(t+2)^{3} 25$ $m m \wedge x(t+1)>0.5 m m \wedge x(t+3)>0.5 m m$, donde $\mathrm{x}(\mathrm{t})$ es la precipitación acumulada en la pentada $t$, con $1 £ \mathrm{t} £ 73$.

Para la determinación del término de la estación lluviosa, o TELL, el proceso de revisión fue idéntico al anterior, pero las pentadas fueron revisadas en orden decreciente; es decir, de la 73 hacia la 1. De los cálculos anteriores, se obtuvieron dos series de tiempo anuales por estación, una del IELL y la otra del TELL. La Fig. 4 muestra la evolución temporal de los valores medios de las estaciones usadas para el IELL y el TELL.
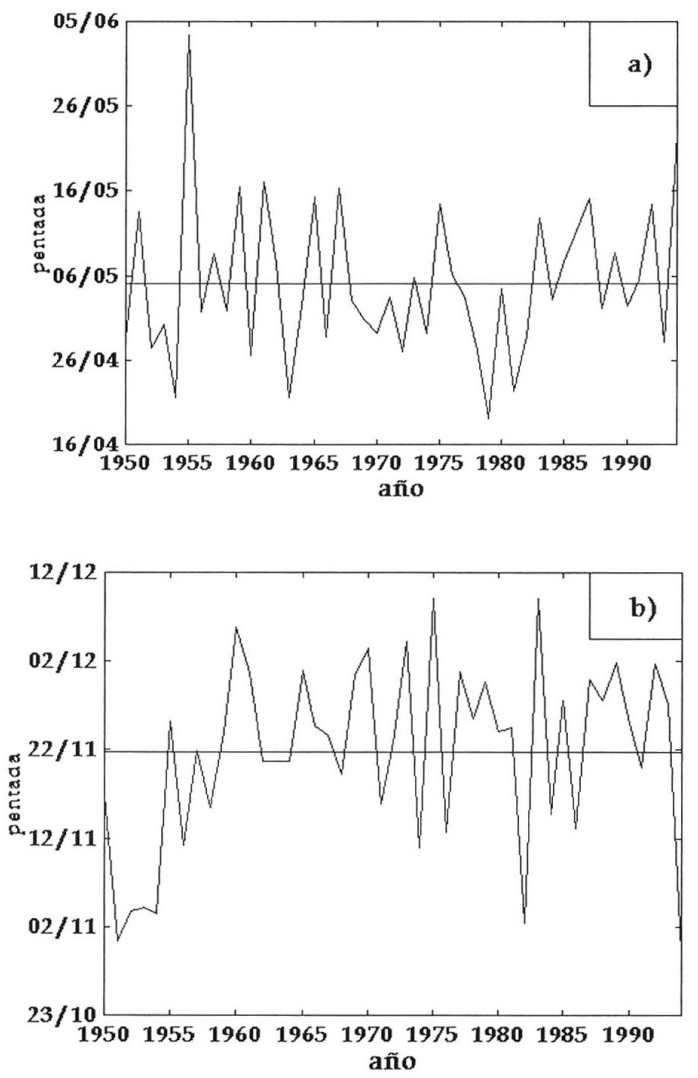

Figura 4. Series temporales medias para a) IELL y b) TELL. La línea horizontal continua denota el promedio de la serie.

Figure 4. Mean time series for a) IELL and b) TELL. The horizontal continous line is the series' average. 
A continuación se produjeron 12 series de tiempo por cada índice (ATN, ATS, Niño3, e IOS); esto es, una por cada mes del año, para obtener la función de correlación cruzada (FCC) de los distintos meses de los índices con los valores de IELL y TELL, para los rezagos anuales de -1 y 0 , donde 0 denota el año para el cual se consideran los índices IELL/TELL y -1 el año anterior a éste. Posteriormente, se reordenaron los valores de la FCC por estación, por índice. La FCC fue calculada para los meses de abril (-1) a marzo (0) para el IELL y de noviembre (-1) a octubre (0) para el TELL. Luego se calculó el promedio de la FCC de todas las estaciones para los meses antes descritos y este promedio se graficó para determinar el mes de máxima correlación entre un índice y la región (Figs. 5, 6 y 7). Este máximo fue tomado en cuenta si al menos era mayor a 1.7s $(a<0.1$ según lo sugerido por Sciremammano, 1979), donde s es el error estándar de la serie.

\section{RESULTADOS Y DISCUSIÓN}

Las correlaciones entre los distintos índices usados se muestran en la tabla 1. Como era de esperar, la más alta se produce entre los índices Niño3 e IOS, pues éstos están asociados con el mismo fenómeno (ENOS), sin embargo, se incluyeron ambos índices en el análisis, pues el primero está más relacionado con la parte oceánica y el segundo con la parte atmosférica del ENOS. Las demás correlaciones son bastantes bajas, al menos para rezagos mensuales iguales a cero.

Tabla 1. Coeficientes de correlación, para los valores mensuales y rezago 0, de los índices usados en el análisis.

Table 1. Correlation coefficients, for monthly data and lag 0 , of the indices used in the analysis.

\begin{tabular}{|lrrrr|}
\hline Indice & ATN & ATS & Niño 3 & IOS \\
\hline ATN & - & 0.02 & -0.16 & 0.05 \\
ATS & 0.02 & - & -0.17 & 0.11 \\
Niño 3 & -0.16 & -0.17 & - & -0.66 \\
IOS & 0.05 & 0.11 & -0.66 & - \\
& & & & \\
\hline
\end{tabular}

La figura 5a indica que la FCC muestra un patrón de correlaciones negativas entre el índice del Atlántico Tropical Norte (ATN) y el IELL(0), para los meses de octubre (-1) a marzo (0), con valores mayores a $1.7 \mathrm{~s}$. Esto se interpreta como que un evento cálido (frío) en esta zona, durante este período del año, coincide con un posterior inicio temprano (tardío) de la estación lluviosa. El mecanismo físico por el cual esto sucede podría deberse a lo siguiente: Estos eventos cálidos están asociados con bajas presiones (relativas al campo medio) en la región del Atlántico Tropical Norte (Hastenrath et al., 1987). Según, Chacón y Fernández (1985), esto disminuye el flujo alisio del Noreste, asociado al anticiclón del Atlántico Norte, sobre Centroamérica $\mathrm{y}$ favorece a su vez el establecimiento sobre la región de la ZCIT, con lo cual se tendrían condiciones propicias para el inicio temprano de la estación lluviosa sobre la región. Condiciones adversas se esperarían para los eventos fríos (Hastenrath et al., 1987). Específicamente, la figura $5 \mathrm{~b}$ muestra la distribución espacial de correlaciones para el mes de enero (0) (con valor promedio mayor a $2.6 \mathrm{~s}$ en la figura 5a). Se notan seis estaciones ubicadas al norte de $12^{\circ} \mathrm{N}$, con correlaciones positivas, que se desvían del patrón general de correlaciones negativas. Por su parte, el índice del Atlántico Tropical Sur (ATS) no mostró correlaciones significativas tanto para el IELL(0) como para el TELL(0).

La figura 6a muestra el comportamiento de la FCC entre el IOS y el TELL(0). En ella se nota un patrón de correlaciones positivas, mayores a $1.7 \mathrm{~s}$, para el período entre noviembre (-1) y febrero (0). Esto nos dice que una fase baja (alta) del IOS, en este período, coincide con un término temprano (tardío) de la estación lluviosa. El mes de máxima correlación fue diciembre (-1), mayor a 2.6s y su distribución espacial se muestra en la figura $6 \mathrm{~b}$. Únicamente la estación Fabio Baudrit, ubicada a $10^{\circ} \mathrm{N}$, sobre el Valle Central de Costa Rica, muestra una correlación negativa no significativa, opuesta al patrón general de anomalías positivas. La figura $7 \mathrm{a}$ muestra un patrón de correlaciones negativas para la FCC entre el índice Niño 3 y el TELL(0) para los meses de noviembre (-1) a marzo (0), mayores a $1.7 \mathrm{~s}$. Este resultado es coherente con el hallado en la figura 6a pero con una diferencia de 3-4 meses entre sus meses de máxima correlación y refleja que un evento cálido (frío) en esta zona oceánica, coincide con un término temprano (tardío) de la estación lluviosa. La razón de lo anterior podría deberse, según Philander (1989), a que estos eventos cá- 

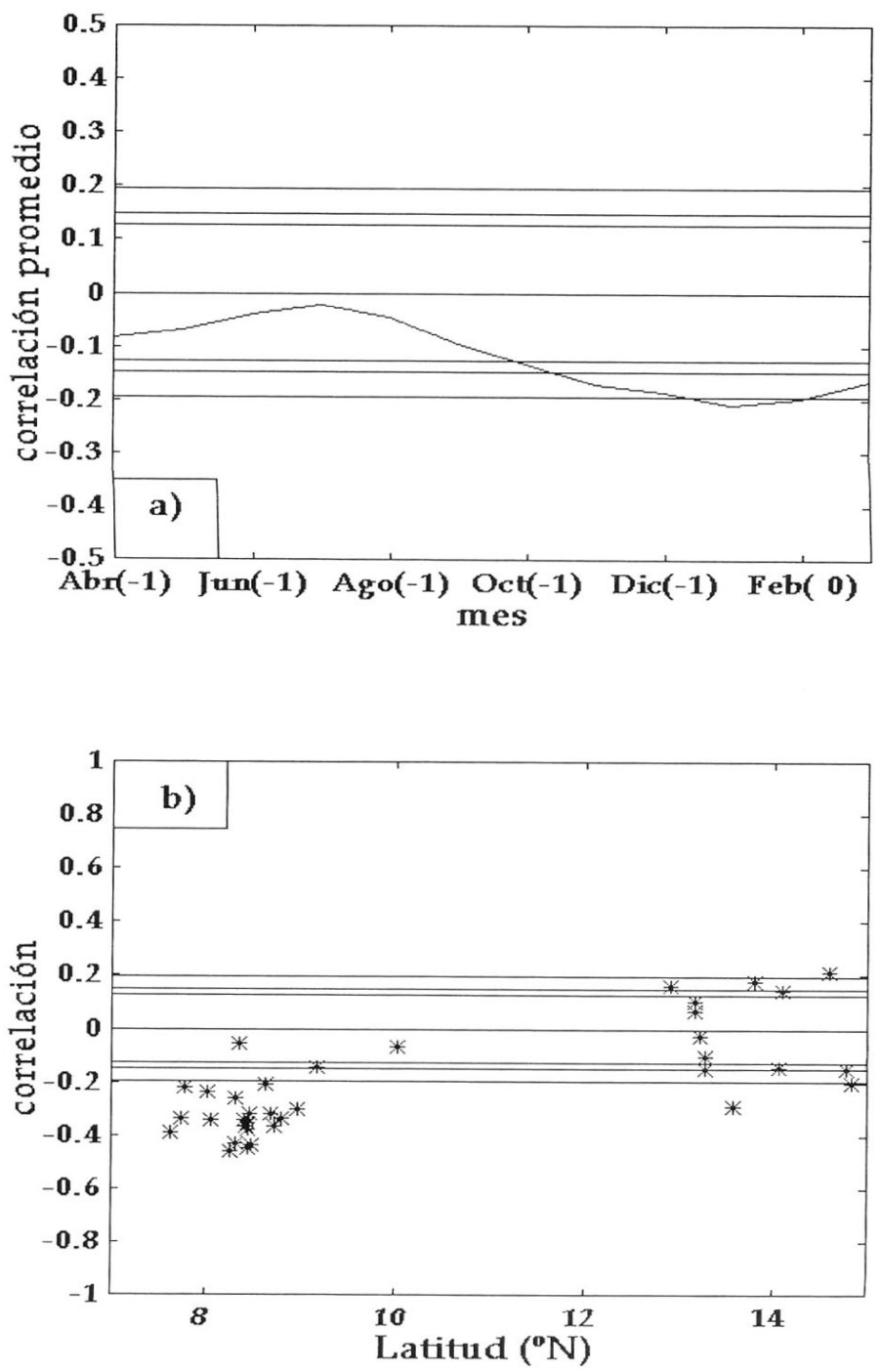

Figura 5. a) Promedio de los coeficientes de la Función de Correlación Cruzada (FCC) entre ATN y IELL(0). Los meses van de Abril(-1) a Marzo(0). b) FFC del mes de Enero(0) en función de la latitud de las estaciones para ATN vs. IELL(0). Los niveles de confianza son para $1.7 \mathrm{~s}, 2 \mathrm{~s}$ y $2.6 \mathrm{~s}$.

Figure 5. a) Average of the Cross Correlation Function (FCC) coefficients for ATN vs. IELL(0). Monthly lags go from April(-1) through $\operatorname{March}(0)$. b) FCC for the month of January (0) for ATN vs. IELL(0). The x axis is the stations' latitude. Confidence levels are given for $1.7 \mathrm{~s}, 2 \mathrm{~s}$ y $2.6 \mathrm{~s}$.

lidos en el Pacífico Ecuatorial Este, favorecen la migración temprana de la ZCIT hacia el sur. Esta migración temprana, está asociada a condiciones secas sobre la región centroamericana, especialmente sobre la Vertiente Pacífica, pues favorece el restablecimiento del dominio del flujo alisio del Noreste (Waylen, 1996b). Por otro lado, durante los eventos fríos, se esperarían condiciones húmedas sobre la región, asociadas a una migración tardía de la ZCIT hacia el sur. La figura 7b muestra la distribución espacial de este patrón de correlaciones para el mes de marzo (0) (mayor que $2 \mathrm{~s}$ según la figura 6a). Únicamente 4 estaciones mostraron correlaciones positivas, no significativas, que se diferencian del patrón general de correlaciones negativas. La diferencia encontrada entre los meses de máxima correlación de los índices IOS y Niño 3 con el TELL(0) está de acuerdo con lo descrito por Enfield 
(1989) para su evento ENOS canónico. Este autor describe una diferencia de 3 a 4 meses entre el colapso de los alisios sobre el Pacífico Ecuatorial
Oriental (parte atmosférica) y la aparición de anomalías positivas frente a las costas de Perú y Ecuador (parte oceánica).
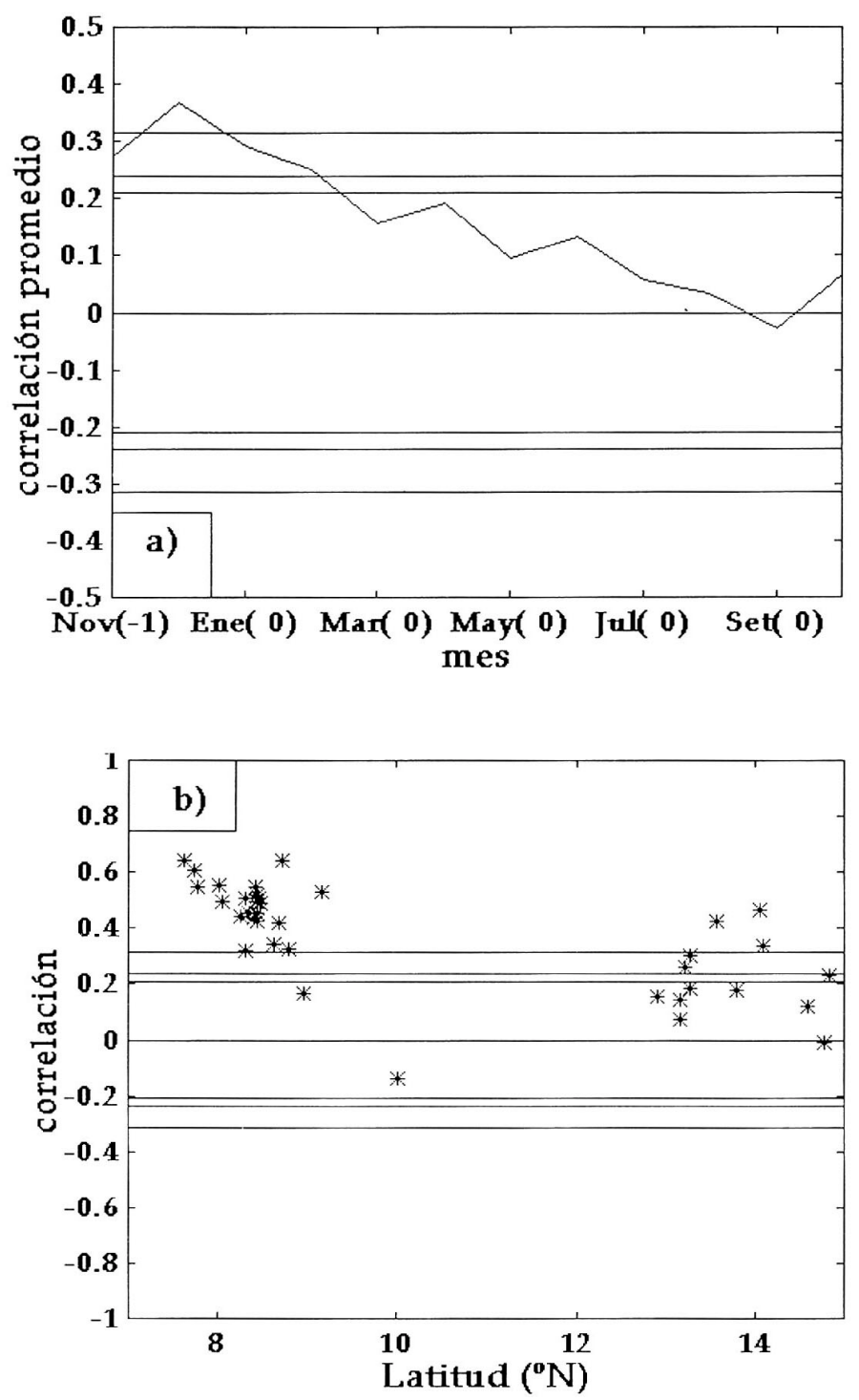

Figura 6. a) Promedio de los coeficientes de la Función de Correlación Cruzada (FCC) entre IOS y TELL(0). Los meses van de Noviembre(-1) a Octubre(0). b) FFC del mes de Diciembre(-1) en función de la latitud de las estaciones para IOS vs. TELL(0). Los niveles de confianza son para $1.7 \mathrm{~s}, 2 \mathrm{~s}$ y $2.6 \mathrm{~s}$.

Figure 6. a) Average of the Cross Correlation Function (FCC) coefficients for SOI vs. TELL(0). Monthly lags go from November(-1) through October(0). b) FCC for the month of December(-1) for SOI vs. TELL(0). The $x$ axis is the stations' latitude. Confidence levels are given for $1.7 \mathrm{~s}, 2 \mathrm{~s}$ y $2.6 \mathrm{~s}$. 

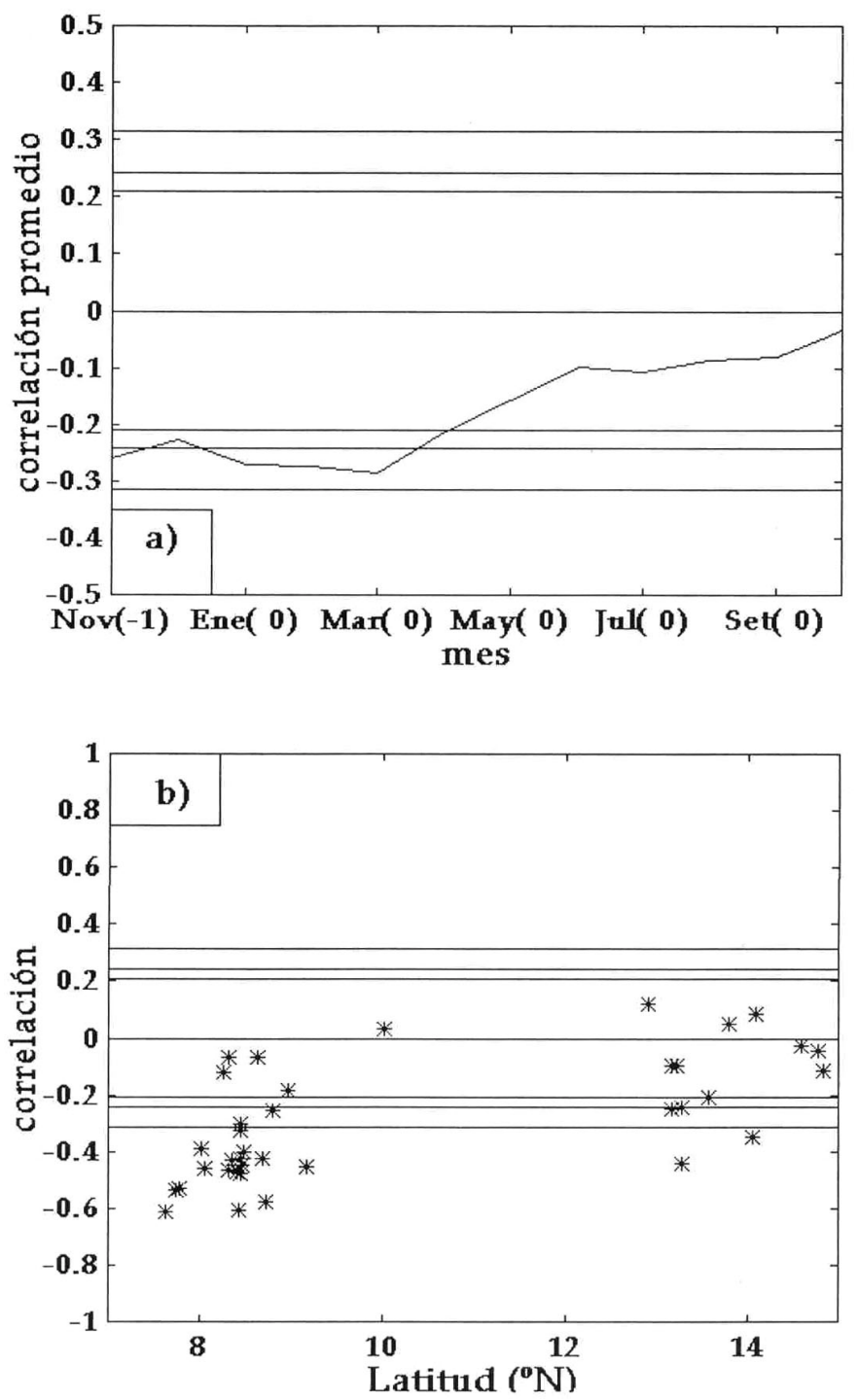

Figura 7. a) Promedio de los coeficientes de la Función de Correlación Cruzada (FCC) entre Niño3 y TELL(0). Los meses van de Noviembre(-1) a Octubre(0). b) FFC del mes de Marzo(0) en función de la latitud de las estaciones para Niño3 vs. TELL(0). Los niveles de confianza son para $1.7 \mathrm{~s}, 2 \mathrm{~s}$ y $2.6 \mathrm{~s}$.

Figure 7. a) Average of the Cross Correlation Function (FCC) coefficients for Niño3 vs. TELL(0). Monthly lags go from November(-1) through $\operatorname{October}(0)$. b) FCC for the month of $\operatorname{March}(0)$ for Niño3 vs. TELL(0). The $x$ axis is the stations' latitude. Confidence levels are given for $1.7 \mathrm{~s}, 2 \mathrm{~s}$ y $2.6 \mathrm{~s}$.

Los resultados anteriores coinciden con los estudios previos de correlación anual y mensual, entre el índice IOS y los campos de precipitación en Centroamérica (e.g. Aceituno, 1988; Hastenrath, 1976, 1978; Kiladis y Díaz, 1989; Ropelewski y Halpert, 1987, 1996; Waylen et al., 1994, 1996a).
Ellos encontraron asociaciones entre los años secos (húmedos) en Centroamérica y los eventos de El Niño (La Niña). Debido a su resolución espacial, este trabajo complementa los resultados encontrados por Enfield (1996) y Enfield y Mayer (1997), debido a que permite ver los efectos sobre la ver- 
tiente Pacífica de Centroamérica. En sus trabajos estos autores encontraron, usando los índices Niño3 y ATN, un incremento de la precipitación sobre la Vertiente Caribe correlacionada con un enfriamiento y/o un calentamiento de las regiones ATN y Niño3 respectivamente. Este incremento de la precipitación es asociado por ellos a un incremento en la intensidad del flujo alisio, lo que a su vez es propuesto por Fernández y Ramírez (1991), Chacón y Fernández (1985), Ramírez (1983) y Waylen et al., (1996b) como causante de sequías en la Vertiente Pacífica.

Por último, las desviaciones del patrón general de algunas estaciones podrían deberse a efectos topográficos locales, los cuales requieren un estudio posterior, pues escapan del alcance de este estudio. Varios autores (e.g. Chacón y Fernández, 1985, Ramírez, 1983, Waylen et al., 1996b), postulan que estas desviaciones anómalas pueden deberse a la existencia de pasos cordilleranos, que permiten la entrada del flujo alisio, transportando humedad hacia la Vertiente Pacífica y que también provocan áreas de surgencia en el Pacífico centroamericano (Umatani y Yamagata, 1991).

\section{CONCLUSIONES}

Únicamente las FCC entre ATN vs. IELL(0), IOS vs. TELL(0) y Niño3 vs. TELL(0) mostraron correlaciones significativas (Fig. 5, 6 y 7), mientras que el índice ATS no mostró correlaciones significativas con el IELL(0) ó el TELL(0).

Las estaciones más sensibles a las variaciones de TSM en las zonas estudiadas, fueron las ubicadas al sur de $10^{\circ} \mathrm{N}$ en la región (Panamá y sur de Costa Rica). A pesar de que los máximos de los valores promedio de la FCC entre los índices y el IELL/ TELL son pequeños en valor absoluto (Figs. 5a, 6a, 7a), ellos fueron mayores al menos a 1.7s y mostraron bastante coherencia espacial (Figs. 5b, 6b, $7 b)$.

El comportamiento del IELL(0) fue dominado por el índice ATN con un patrón de correlaciones negativas con máximo en enero (0), lo que sugiere que un evento cálido (frío) se correlaciona con un comienzo temprano (tardío) de la estación lluviosa. El comportamiento del TELL(0) fue dominado por los índices IOS y Niño3 con un patrón de correlaciones positivas y negativas en diciembre (-1) y marzo (0) respectivamente. Esto sugiere que un evento cálido (frío) en el Pacífico Ecuatorial Este se correlaciona con un comienzo temprano (tardío) de la estación seca. Esta relación puede ser entendida dentro del desarrollo del ENOS canónico propuesto por Rasmuson y Carpenter (1982). Ellos postulan anomalías positivas de TSM durante casi todo el año(0), pero sitúan el máximo del evento a principios de ese mismo año. Los resultados anteriores confirman y complementan estudios anteriores que relacionaron el ENOS, a través del IOS, con la precipitación en Centroamérica pero después de iniciada la estación lluviosa (e.g. Aceituno, 1988; Kiladis and Díaz, 1989; Rogers, 1988; Ropelewski and Halpert, 1987, 1989; Waylen et al., 1994).

En resumen, este trabajo revela que el comportamiento anómalo de la estación lluviosa en Centroamérica no está influenciado por una sola área oceánica sino más bien por dos, el Atlántico Tropical Norte y el Pacífico Tropical Este, que a su vez dominan distintos aspectos de la estación lluviosa, como son el comienzo y el término de la misma, respectivamente.

\section{AGRADECIMIENTOS}

Este trabajo se realizó gracias a los fondos del proyecto P.I. 96.112.039-6, Universidad de Concepción. Los datos de precipitación fueron obtenidos del banco de datos del CIGEFI, UCR y del IMN de Costa Rica. Los datos de TSM fueron cedidos gracias a la participación en este trabajo del Dr. David Enfield, AOML, NOAA, dentro del marco del proyecto TC3, IAI. Los autores agradecen al Dr. Jorge Amador, UCR, por su valiosa participación en la discusión de la metodología empleada.

\section{REFERENCIAS}

Aceituno, P., 1988. On the functioning of the Southern Oscillation in the South American sector. Part I: Surface Climate. Mon. Weather Rev., 116: 505524.

Chacón, E., y W. Fernández, 1985. Temporal and spatial rainfall variability in the mountain region of the Reventazón River basin, Costa Rica. J. Climate., 5, 175-188.

Díaz, H. y G. Kiladis, 1993. Atmospheric teleconnections associated with the extreme phase of the Southern Oscillation. In: H. Díaz y V. 
Markgraf (Ed.) El Niño. Historical and Paleoclimatic Aspects of the Southern Oscillation. Cambrige University Press, pp. 7-28.

Enfield, D. 1989. El Niño, past and present. Rev. Geophys., 27 (1): 159-187.

Enfield, D., 1996. Relationships of inter-American rainfall to tropical Atlantic and Pacific SST variability. Geophys. Res. Lett., 23(23): 33053308 .

Enfield, D., y D. Mayer, 1997. Tropical Atlantic sea surface temperature variability and its relation to El Niño-Southern Oscillation. J. Geophys. Res., 102(C1): 929-945.

Fernández, W., y P. Ramírez, 1991. El Niño, La Oscilación del Sur y sus efectos en Costa Rica: una revisión. Tecnol. Marcha, 11: 3-11.

Gray, C., 1993. Regional meteorology and Hurricanes. In: G. Maul (Ed.) Climatic Change in the IntraAmericas Sea. Eduard Arnold, pp. 87-99.

Hastenrath, S., 1976. Variations in low-latitude circulation and extreme climatic events in the Tropical Americas. J. Atmos. Sci., 33: 202-215.

Hastenrath, S., 1978. On modes of tropical circulation and climate Anomalies. J. Atmos. Sci., 35, 22222231.

Hastenrath, S., L. de Castro y P. Aceituno, 1987. The Southern Oscillation in the Atlantic sector. Contrib. Atmos. Phys., 60: 447-463.

Kiladis, G. y H. Díaz, 1989. Global climatic anomalies associated with extremes in the Southern Oscillation. J. Climate, 2: 1069-1090.

Philander, G., 1989. El Niño and La Niña. Am. Sci., September-October, 451-459.

Ramírez, P., 1983. Estudio meteorológico de los veranillos en Costa Rica. Nota de investigación $\mathrm{N}^{\circ}$ 5: Instituto Meteorológico Nacional, Ministerio de Agricultura y Ganadería. San José, Costa Rica.

Rasmuson, E. y T. Carpenter, 1982. Variations in tropical sea surface temperature and surface wind fields associated with the Southern Oscillation/El Niño. Mon. Weather Rev., 110: 354-384.

Recibido el 26 de mayo de 1997. Aceptado el 14 de mayo de 1998.
Rogers, J., 1988. Precipitation variability over the Caribbean and tropical Americas associated with the Southern Oscillation. J. Climate, 1: 172-182.

Ropelewski, C. y M. Halpert, 1987. Global and regional scale precipitation associated with $\mathrm{El} \mathrm{Nino/}$ Southern Oscillation. Mon. Weather Rev, 115: 1606-1626.

Ropelewski, C. y M. Halpert, 1989. Precipitation patterns associated with the high index phase of the Southern Oscillation. J. Climate, 2: 268-284.

Ropelewski, C. y M. Halpert, 1996. Quantifying Southern Oscillation-precipitation relationships. J. Climate, 9: 1043-1059.

Ropelewski, C. y P. Jones, 1987. An extention of the Tahiti-Darwin Southern Oscillation Index. Mon. Weather Rev., 115: 2161-2165.

Sciremammano, F., 1979. A suggestion for the presentation of correlations and their significance levels. J. Phys. Oceanogr., 9: 1273-1276.

Smith, T., R. Reynolds, R. Livezy y D. Stokes, 1996. Reconstruction of historical sea surface temperatures using empirical orthogonal functions. J. Climate, 9: 1403-1420.

Umatani, S. y T. Yamagata, 1991. Response of the Eastern Tropical Pacific to meridional migration of the ITCZ: The generation of the Costa Rica Dome. J. Phys. Oceanogr., 21: 346-363.

Waylen, P., M. Quesada y C. Caviedes, 1996a. Temporal and spatial variability of annual precipitation in Costa Rica and the Southern Oscillation. Int. J. Climatol., 16: 173-193.

Waylen, P., C. Caviedes y M. Quesada, 1996b. Interannual variability of monthly precipitation in Costa Rica. J. Climate, 9: 2606-2613.

Waylen, P., M. Quesada y C. Caviedes, 1994. The effects of El Niño-Southern Oscillation on precipitation in San Jose, Costa Rica. Int. J. Climatol., 14: 559-568.

Woodruff, S., R. Slutz, R. Jenne y P. Steurer, 1987. A comprehensive Ocean-Atmosphere data set. Bull. Amer. Meteorol. Soc., 68(10): 1239-1250.

Yao, A., 1981. Agricultural climatology. In: E. Landsberg (Ed.) World Survey of Climatology, vol. 3. Elsevier Scientific Publishing Company, pp. 189-298. 